\title{
Tagung: „Was willst du, dass ich dir tun soll?“ Ethik in Seelsorge und Spiritual Care
}

\author{
15. und 16. September 2021 in Zürich
}

\author{
Gwendolin Wanderer · Sebastian Farr · Florian-Sebastian Ehlert
}

Angenommen: 11. November 2021 / Online publiziert: 14. Dezember 2021

(C) Der/die Autor(en) 2021

Im Zuge einer zunehmenden Professionalisierung der Medizinethik wie auch der Seelsorge und im Zusammenhang mit der Einbindung von Seelsorge in die interprofessionelle Spiritual Care, rückt seit einiger Zeit die Frage nach dem ethischen Profil und der ethischen Qualifikation von Seelsorge stärker in den Blick auch der Forschung. Empirische Untersuchungen der letzten Jahre haben auf zahlreiche Berührungspunkte zwischen Seelsorge, Spiritual Care und Ethik aufmerksam gemacht, die auch über die strukturierte Einbindung von Seelsorgenden in Entscheidungsprozesse bei ethischen Fragestellungen hinausgehen. Seelsorgende sind somit in ihrer praktischen Tätigkeit herausgefordert, ethische Kompetenzen für das Praxisfeld der klinischen Versorgung auszubilden und im Verhältnis zu ihrer Rolle als Seelsorgende zu reflektieren. Die Tagung in Zürich greift den Bedarf an wissenschaftlichem Austausch und Vernetzung auf und steht im weiteren Zusammenhang mit der Arbeitsgruppe Ethik in Seelsorge und Spiritual Care in der Akademie für Ethik in der Medizin. Im Rahmen der Hauptvorträge wurden einschlägige Forschungsarbeiten im Bereich der Ethik in Seelsorge und Spiritual Care vorgestellt und miteinander ins Gespräch gebracht.

Dr. Gwendolin Wanderer $(\bowtie)$

Arbeitsstelle Medizinethik in der Klinikseelsorge, Goethe-Universität Frankfurt (FB07),

Norbert-Wollheim-Platz 1, 60323 Frankfurt am Main, Deutschland

E-Mail: g.wanderer@em.uni-frankfurt.de

Sebastian Farr

Institut für Sozialethik, Zürich, Schweiz

Florian-Sebastian Ehlert

Arbeitsstelle Ethik im Gesundheitswesen, Kirchenkreisverband Hamburg, Hamburg, Deutschland 


\section{Konzeptionen von Spiritual Care und ethische Fragestellungen}

Simon Peng-Keller (Professor für Spiritual Care/Univ. Zürich) leitet mit seinem Vortrag in die Tagung mit einer Darstellung unterschiedlicher Konzeptionen von Spiritual Care im deutschsprachigen Raum ein und weist darauf hin, dass eine Engführung der Definition unsachgemäß ist. Stattdessen bietet er folgendes Gliederungsmodell an:

1. Klinisches Modell: Leitidee der Erweiterung des biopsychosozialen Ansatzes um eine ,spirituelle“ Dimension.

a. Spiritual Care findet im Behandlungsteam im Gegenüber zur kirchlichen Seelsorge statt.

b. Spiritual Care als eine gemeinsame interprofessionelle Aufgabe (einschließlich der Seelsorge).

c. Unterscheidung zwischen allgemeiner Spiritual Care des Gesundheitswesens und spezialisierter Spiritual Care der Seelsorge.

2. Spiritual Care als gemeinschaftsbezogenes Modell einer zivilgesellschaftlichen Aufgabe (caring communities).

3. Holistisches Modell eines umfassenden Heilwerdens (healing vs. cure).

Im Sinne einer spezialisierten Spiritual Care (1c) erhält Seelsorge damit als eigenständige Profession im Gesundheitswesen eine gesonderte Aufgabe mit ethischen Anschlussfragen. In ihrer Professionsethik sind Seelsorgende zwei Ethiken verpflichtet: Zum einen den ethischen Ansprüchen der Organisationen, in denen sie tätig sind; zum anderen den ethischen Ansprüchen der Glaubensgemeinschaft, der sie angehören. Sie haben des Weiteren die Aufgabe, über Zielsetzungen, Handeln und Qualitätssicherung Rechenschaft abzulegen. Hieran schließt Peng-Keller die Diskussion einer therapeutischen Orientierung der Seelsorge an: Dass Seelsorge keine Therapien anbietet, bedeutet nicht, dass sie nicht therapeutisch wirksam sein und in einem interprofessionellen Kontext zu palliativen, kurativen, rehabilitativen und präventiven Zielsetzungen beitragen kann.

Offen bleibt, ob und inwieweit eine spirituelle Verwurzelung als Voraussetzung spezialisierter Spiritual Care einforderbar ist. Gleichzeitig kann jedoch überprüft werden, wie offen jemand über seine spirituellen Hintergründe spricht; wie diese Hintergründe und Kompetenzen zu den Bedürfnissen der Patient:innen und der Institution passen und wie weit jemand fähig ist, sich auf fremde Überzeugungen/ Praktiken/Erfahrungen einzulassen.

\section{Die Bedeutung von Seelsorge und Spiritual Care aus der Perspektive der Medizinethik}

Laut Christoph Rehmann-Sutter (Philosoph und Bioethiker/Univ. zu Lübeck) weist die Adressierungsstruktur der Ethik über die Reichweite von biomedizinischen Beschreibungen und Urteilsakten hinaus. Er beschreibt die ethische Beziehung als eine Frage nach dem Du: Dem Du als dem ganz Anderen, als dem, das die eigene Selbstgewissheit in Frage stellt, das als eine eigene Narration verstanden werden kann. 
Aus der Begegnung mit dem „Du“ folgt immer auch eine Adressierungsstruktur, die ein „Sollen“ ergibt, das in konkreten Situationen zum Ausdruck kommt.

Zusammenfassend formuliert er: „Die Frage, ob und wie Ethik in der Lage ist, spirituelle Dimensionen in die Klärung ihrer Fragen aufzunehmen, entscheidet sich nicht auf der Ebene der ethischen Methoden und Ansätze, sondern auf einer fundamentaleren Ebene: bei der Adressierungsstruktur der Ethik.“

„Im Zentrum steht die Position des:der Anderen in ihrer Lebensgeschichte. Zusammen mit den medizinischen Aspekten bildet sie die Situation aus, aus der heraus sich ein Sollen ergibt und aus der heraus sich ein Dilemma erst verstehen lässt.“ Ethik ist damit auf Narrationen angewiesen, die die jeweiligen Adressierungen begründen und illustrieren. Letztlich sollte eine Ethik der Spiritualität eine hörende Ethik sein, die zu verstehen sucht.

\section{Medizinethik in der Klinikseelsorge - Themen, Methoden, Kontexte}

Hille Haker (theologische Ethikerin/Loyola Univ., Chicago) skizziert in ihrem Vortrag die Entwicklung der Professionalisierung der Klinikseelsorge seit den siebziger Jahren. Durch die Clinical Pastoral Education - in Deutschland durch die Klinische Seelsorgeausbildung (KSA) - wurde ein Professionalisierungsschub erreicht. Seelsorge erfuhr mehr und mehr Einbindung in das medizinische Team wie auch in institutionalisierte klinische Ethikgremien. Sie versteht sich als Begleitung in existentiellen Krisen und macht sich zur Aufgabe, sich für die Wahrung und Wiederherstellung der Würde von Patient:innen einzusetzen. Haker zeigt auf, dass damit letztendlich alle Themen der Medizinethik auch Themen einer Klinikseelsorgeethik sind. Eine Kategorisierung der methodischen Kontexte der Klinikseelsorgeethik: 1.) Selbstsorge/Fürsorge (Care), 2.) soziale und gemeinschaftliche Werte/soziale Normen, 3.) Pflichten, Rechte und Verantwortung und 4.) Institutionen, Recht und Gerechtigkeit hilft, diese systematisch in den Blick zu nehmen.

Zentral sind für Haker dabei unter anderem folgende Aspekte: 1.) die Erfahrung von Brüchen, Leiden und Isolation, der durch die Vermittlung von Anerkennung, Trost und Beziehung zu begegnen ist, 2.) Solidarität und Zugehörigkeit, 3.) Menschenwürde und verletzliche Freiheit und 4.) Gerechtigkeit.

Verletzlichkeit als Prinzip, das heißt als Prinzip der Freiheit eingedenk der Verletzlichkeit des Menschen, ist für eine Weiterentwicklung der Klinikseelsorgeethik besonders relevant. Diese vermag es nämlich, die Leiblichkeit des Menschen - die auch die Affizierbarkeit des zum Helfen Aufgerufenen meint - mit der Pflicht zur Ermöglichung der Menschenwürde als Freiheit zusammenzubringen.

\section{Vulnerabilität als Thema von Seelsorge und Ethik}

Andrea Biehler (Praktische Theologin/Univ. Basel) beschreibt Vulnerabilität als einen Seelsorge und Ethik verbindenden Begriff. Allerdings tragen Seelsorge und Ethik unterschiedliche Blickwinkel und Herangehensweisen an dieses Phänomen heran. Sie führt die Verletzlichkeit des Menschen fundamental als zum Menschen ge- 
hörend (Vulnerabilität als conditio humana) ein: Dem Menschen kommt eine grundlegende Offenheit in Beziehungen zu, dazu gehört die Berührbarkeit/Affizierbarkeit. Zur Arbeit mit Vulnerabilität gehört auch die Reflexion der vieldimensionalen Abhängigkeitsverhältnisse. Nach einem Diskurs über Aspekte von Verletzlichkeit in der Theologie (Stichworte: Altern, Verletzlichkeit Gottes, Diakonie) differenziert sie verschiedene Formen zwischen fundamentaler und situativer Vulnerabilität, wobei in beiden sowohl Aspekte von Leiblichkeit, aber ebenso auch politische Diskurse eine Rolle spielen. Die Seelsorge kommt in Momenten des Leidens ins Spiel, welches Biehler als eine Fremdheitserfahrung des Leibes und als physische Erfahrung des Negativen bestimmt. Ausgehend von der These „Gott als Wirklichkeit des Möglichen“ trägt Seelsorge die „Lebenskunst, unter gegebenen Bedingungen ein nicht vorgegebenes Leben zu führen“ (W. Engemann) in die Begegnungen ein.

In der Reflexion über ,,verletzliche Freiheit“ kommen Ethik und Seelsorge wieder in einem sie verbindenden Diskurs zusammen. In diesem Diskurs fordert Biehler eine moralische Grundentscheidung ein: nämlich zu differenzieren, was sozial konstruiert und was ontologisch gesetzt ist - d.h. zu differenzieren zwischen Auflehnung und Hinnahme. Aufgabe von Ethik und Seelsorge ist es, diese Frage kritisch zu reflektieren.

\section{Seelsorge und schwierige Entscheidungen am Lebensende - Beobachtungen vor dem Hintergrund der Resultate des NFP 67 ,Lebensende ${ }^{66}$}

In seinem Vortrag stellt Markus Zimmermann (theologischer Ethiker/Univ. Freiburg) die These auf, dass Seelsorgende und klinische Ethiker:innen unterschiedliche Rollen in einem Krankenhaus haben und sich in ihrer Arbeit auf unterschiedliche Expertisen und Erfahrungen stützen. Mit Walter Lesch betont Zimmermann, dass es in der Bearbeitung ethischer Fragen im Krankenhaus um eine „Interdisziplinarität ohne Disziplinlosigkeit“ gehe bzw. gehen solle. Anhand von Resultaten der (Schweizer) Nationalen Forschungsgruppe „Lebensende“ (NFP 67) zeigt Zimmermann auf, dass Zugänge zu Fragen des ,guten Sterbens“ aus den Bereichen Spiritual Care und Seelsorge unterschiedliche Sinngehalte von Sterbewünschen und -narrativen besonders aufdecken. Dem gegenüber stehe eine ,medizinisierte Medizinethik“, die aufgrund ihrer impliziten medizinischen Zugänge von einer verdeckten Normativität gekennzeichnet sei - dies verenge die Wahrnehmung der Sinnfragen am Lebensende. Er belegt dies anhand unterschiedlicher Präferenzen der ärztlichen Therapien am Lebensende in unterschiedlichen geographischen Räumen.

Seelsorgende, die in die Begleitung von Menschen am Lebensende in besonderer Weise involviert sind, haben in der Regel eine kritische Distanz zur Organisation Krankenhaus und zu den dazugehörenden Entscheidungsprozessen. Sie sind eher subversiv und einseitig patientenorientiert (ausgerichtet auf Sinnfragen und nicht ausschließlich auf Behandlung). In dieser Rolle haben sie eine besondere Bedeutung und sollten ihre Expertise schwerpunktmäßig in die Begleitung Sterbender einbringen und gegebenenfalls deren Interessen, Ansichten und Ideale verbalisieren. Seelsorge beraubt sich ihrer Potenziale, wenn sie zu medizinethisch wird. 


\section{Wenn der Rat der Seelsorge gefragt ist - Zum Rollenverständnis der Seelsorge zwischen Spiritual Care und Ethik}

Nach Traugott Roser (Praktischer Theologe/Westfälische Wilhelms-Univ., Münster) stellt sich die Frage nach dem Rollenverständnis der Seelsorge zwischen pastoraler Zuwendung und Unterstützung in ethischen Fragen angesichts der wieder neu virulent gewordenen Diskussion um den ärztlich assistierten Suizid nach dem Urteil des Bundesverfassungsgerichts im Februar 2020 mit großer Dringlichkeit. Im Anschluss an den Artikel in der FAZ von Anselm, Lilie und Karle greift Roser diese Kontroverse auf und erwägt: Der Seelsorge könnte in diesem Feld die Aufgabe einer ,erweiterten Kasualpraxis“ zukommen. Bezogen auf die Ebene des Einzelfalls gestalten Seelsorgende also den Lebensweg von Personen mit und geben ihm im jeweiligen Sinne einen religiösen Rahmen, beteiligen sich an der Bearbeitung und Bewältigung des fragilen Lebens. Seelsorge ist jedoch nicht nur auf der Einzelfallebene tätig, sondern kann auch direkt in die Prozesse der Entwicklung einer ,ethischen Haltung“ eines Unternehmens (z. B. in diakonisch getragenen Einrichtungen) einbezogen werden. Sie übernimmt damit Aufgaben mit struktureller Bedeutung, die weit über anwaltschaftliches Handeln für Patient:innen hinausgehen.

Die Wirksamkeit der Seelsorge in ethischen Konfliktsituationen - verstanden als aktuell subjektiv empfundene Vulnerabilität - liegt für Roser in einer besonderen Sensibilität der Seelsorgenden für Vulnerabilität, dem Raumgeben für solche grundmenschlichen Erfahrungen und die Aufnahme dieser Erfahrungen in religiösen Formen.

Die Hauptvorträge der Tagung wurden durch die Vorstellung von laufenden Forschungs- und Praxisprojekten in parallelen Sektionen ergänzt, die sich inhaltlich mit verschiedenen Teilbereichen der Arbeit der Klinikseelsorge (z. B. Psychiatrieseelsorge, Seelsorge in der Kinderklinik), Querschnittsthemen wie der Frage nach der Dokumentation der erfolgten Seelsorgebesuche, grundlegenden Fragen der Rolle der Klinikseelsorge im ethischen Feld sowie der Entwicklung der muslimischen Seelsorge beschäftigten.

Die Fachtagung hat im Diskurs einen Beitrag geleistet, indem sie Wissenschaftler:innen und Praktiker:innen zusammengebracht hat, um damit Impulse für die weitere Entwicklung von Ethik-Kompetenz in Seelsorge und Spiritual Care im deutschsprachigen Raum sowie für die internationale Forschung zu setzen. Insbesondere die Bedeutung der „Vulnerabilität“ als anthropologische Grunddimension sowie zentraler Inhalt der Seelsorge wurde während der Tagung als verbindendes Element der einzelnen Fachvorträge und Praxisprojektvorstellungen immer wieder ersichtlich. Deutlich geworden ist zudem ein Spannungsverhältnis der Themenfelder, die sich in streitbaren Thesen manifestiert haben. Die Bandbreite reichte dabei von Abschied der Seelsorge von einer medizinisierten klinischen Ethik bis zur Integration der ethischen Großtheorien in die Klinikseelsorgeethik. Die Stellung von Spiritual Care innerhalb der klinischen Ethik verlangt als offene Frage der weiteren Bearbeitung im Austausch von Forschung und Praxis.

Funding Open Access funding enabled and organized by Projekt DEAL. 
Open Access Dieser Artikel wird unter der Creative Commons Namensnennung 4.0 International Lizenz veröffentlicht, welche die Nutzung, Vervielfältigung, Bearbeitung, Verbreitung und Wiedergabe in jeglichem Medium und Format erlaubt, sofern Sie den/die ursprünglichen Autor(en) und die Quelle ordnungsgemäß nennen, einen Link zur Creative Commons Lizenz beifügen und angeben, ob Änderungen vorgenommen wurden.

Die in diesem Artikel enthaltenen Bilder und sonstiges Drittmaterial unterliegen ebenfalls der genannten Creative Commons Lizenz, sofern sich aus der Abbildungslegende nichts anderes ergibt. Sofern das betreffende Material nicht unter der genannten Creative Commons Lizenz steht und die betreffende Handlung nicht nach gesetzlichen Vorschriften erlaubt ist, ist für die oben aufgeführten Weiterverwendungen des Materials die Einwilligung des jeweiligen Rechteinhabers einzuholen.

Weitere Details zur Lizenz entnehmen Sie bitte der Lizenzinformation auf http://creativecommons.org/ licenses/by/4.0/deed.de.

\section{Einhaltung ethischer Richtlinien}

Interessenkonflikt G. Wanderer, S. Farr und F.-S. Ehlert geben an, dass kein Interessenkonflikt besteht.

Ethische Standards Für diesen Beitrag wurden von den Autor:innen keine Studien an Menschen oder Tieren durchgeführt. Für die aufgeführten Studien gelten die jeweils dort angegebenen ethischen Richtlinien. 